International Journal of Applied Mathematical Research, 1 (3) (2012) 342-354 (C) Science Publishing Corporation

www.sciencepubco.com/index.php/IJAMR

\title{
Bi-Level Multi-Objective Absolute-Value Fractional programming Problems: A Fuzzy Goal Programming approach
}

\author{
Mansour Saraj and Sadegh Sadeghi \\ Faculty of Mathematical Sciences and Computer \\ Shahid Chamran University, Ahvaz- Iran \\ Email:msaraj@scu.ac.ir \\ Faculty of Mathematical Sciences and Computer \\ Shahid Chamran University, Ahvaz- Iran \\ Email:s-sadeghi@mscstu.scu.ac.ir
}

\begin{abstract}
In this paper we propose a fuzzy goal programming method for obtaining a satisfactory solution to a bi-level multi-objective absolutevalue fractional programming (BLMO-A-FP) problems. In the proposed approach, the membership functions for the defined fuzzy goals of all objective functions at the two levels as well as the membership functions for vector of fuzzy goals of the decision variables controlled by upper level decision maker (ULDM) are developed in the model formulation of the problem. Then fuzzy goal programming technique is used for achieving highest degree of each of the membership goals by minimizing negative and positive deviational variables. The method of variable change on the under- and over-deviational variables of the membership goals associated with the fuzzy goals of the model is introduced to solve the problem efficiently by using linear goal programming methodology. Theoretical results is illustrated with the help of a numerical.
\end{abstract}

Keywords: Bi-level programming, Multi-Objective, Fractional programming, Goal programming, Fuzzy goal programming, Absolute value.

Mathematics Subject Classification: 90C29; 90C32; 90C70. 


\section{Introduction}

The concept of the Bi-level programming problem (BLPP) was first introduced by Candler and Townsley [1] in 1982. Bi-level programming problem is a special case of a multilevel programming problem (MLPP) of a large hierarchical decision system. In a BLPP, two decision makers (DMs) are located at two different hierarchical levels, each independently controlling one set of decision variables and with different and perhaps conflicting objectives.

In the hierarchical decision process, the lower-level DM (LLDM) executes his/her decision powers, after the decisions of the upper-level DM (ULDM). Although the ULDM independently optimizes its own benefits, the decision may be affected by the reaction of the LLDM. As a consequence, decision deadlock arises frequently and the problem of distribution of proper decision power is encountered in most of the practical decision situations.

Fuzzy goal programming (FGP) is an extension of the conventional goal programming (GP) introduced by Charnes and Cooper [2] in 1961. As a robust tool for MODM problems, GP has been studied extensively in [3] for the last 35 years. In the recent past, FGP in the form of classical GP has been introduced by Mohamed [4] and further studied in [5, 6].

Abo-Sinha [7] discussed multi-objective optimization for solving non-linear multi-objective bi-level programming problem in fuzzy environment. Baky [8] studied FGP algorithm for solving decentralized bi-level multi-objective programming problems. In this study, we formulated FGP algorithm for solving a bi-level multi-objective fractional programming problems with absolute-value functions. A bi-level multi-objective absolute-value fractional programming problems involves a single decision maker viz. upper level decision maker with multi-objectives at the upper level and a single decision maker viz. lower level decision maker with multiple objectives at the lower level. The objective functions of each level decision maker are absolute-value in natural and the system constraints are linear functions.

\section{Problem Formulation}

Let both the ULDM and the LLDM have a motivation to cooperate with each other and try to minimize his/her own benefit, paying serious attention to the preferences of the other. Then, the vectors of decision variables $X_{1}=$ $\left(x_{1}^{1}, x_{1}^{2}, \ldots, x_{1}^{n_{1}}\right)$ and $X_{2}=\left(x_{2}^{1}, x_{2}^{2}, \ldots, x_{2}^{n_{2}}\right)$ where $n=n_{1}+n_{2}$, are under the control of the ULDM and LLDM, respectively. Also we assume that

$$
F_{i}\left(X_{1}, X_{2}\right): R^{n_{1}} \times R^{n_{2}} \longrightarrow R^{m_{i}} \quad i=1,2,
$$

be their respective differentiable absolute-value preference functions. Such a BLMO-A-FP problem of minimization type can be presented as [8] 
(Upper Level)

$$
\min _{X_{1}} F_{1}\left(X_{1}, X_{2}\right)=\min _{X_{1}}\left(f_{11}\left(X_{1}, X_{2}\right), f_{12}\left(X_{1}, X_{2}\right), \ldots, f_{1 m_{1}}\left(X_{1}, X_{2}\right)\right),
$$

where $X_{2}$ solves

(Lower Level)

$$
\min _{X_{2}} F_{2}\left(X_{1}, X_{2}\right)=\min _{X_{2}}\left(f_{21}\left(X_{1}, X_{2}\right), f_{22}\left(X_{1}, X_{2}\right), \ldots, f_{2 m_{2}}\left(X_{1}, X_{2}\right)\right),
$$

subject to

$$
X \in S=\left\{X=\left(X_{1}, X_{2}\right) \in R^{n} \mid A_{1} X_{1}+A_{2} X_{2}\left(\begin{array}{c}
\leq \\
= \\
\geq
\end{array}\right) b, b \in R^{m}\right\} \neq \emptyset
$$

Here

$$
f_{i j}\left(X_{1}, X_{2}\right)=\frac{\alpha_{i j}+\sum_{k=1}^{n} c_{i k}\left|x_{k}\right|}{\beta_{i j}+\sum_{k=1}^{n} d_{i k}\left|x_{k}\right|}, \quad i=1,2, \quad j=1,2, \ldots, m_{i},
$$

where $X$ is unrestricted, $m_{i}(i=1,2)$ are the number of DMs objective functions, $m$ is the number of the constraints, $\alpha_{i j}$ and $\beta_{i j}\left(i=1,2, \quad j=1,2, \ldots, m_{i}\right)$ are the scalars, $A_{1}$ and $A_{2}$ are constant matrices, $c_{i k}$ and $d_{i k}(i=1,2, \quad k=$ $1,2, \ldots, n)$ are unconstrained in sign, without loss of generality it is customary to assume that $\beta_{i j}+\sum_{k=1}^{n} d_{i k}\left|x_{k}\right|>0$. Also we assume that $\overline{l_{i j}} \leq f_{i j} \leq \overline{u_{i j}}$ $\left(i=1,2, \quad j=1,2, \ldots, m_{i}\right)$ where $\overline{l_{i j}}$ and $\overline{u_{i j}}$ are, respectively, upper and lower bounded of $f_{i j}\left(X_{1}, X_{2}\right)$.

\section{Formulation of the FGP Problem}

In BLMO-A-FP, if an imprecise aspiration level is assigned to each of the objectives, then the fuzzy objectives are termed as fuzzy goals.

The solutions usually are different because of conflicts of nature between two objectives. Therefore, it can easily be assumed that all values larger than or equal to $\overline{u_{i j}}\left(i=1,2, \quad j=1,2, \ldots, m_{i}\right)$ are absolutely unacceptable to leader and follower, respectively. So $\overline{u_{i j}}$ can be considered as the upper tolerance limits of the respective fuzzy objective goals. Then, membership functions $\mu_{f_{i j}}\left(f_{i j}\left(X_{1}, X_{2}\right)\right.$ for the $i j$ th fuzzy goal can be formulated as

$$
\mu_{f_{i j}}\left(f_{i j}\left(X_{1}, X_{2}\right)=\left\{\begin{array}{cr}
1 & f_{i j} \leq \bar{l}_{i j} \\
\frac{\bar{u}_{i j}-f_{i j}\left(X_{1}, X_{2}\right)}{\bar{u}_{i j}-\bar{l}_{i j}} & \bar{l}_{i j} \leq f_{i j} \leq \bar{u}_{i j} \\
0 & f_{i j}>\bar{u}_{i j}
\end{array}\right.\right.
$$

To build the membership functions for the fuzzy goals of the decision variables controlled by ULDM, the optimal solution $X^{*}=\left(X_{1}^{*}, X_{2}^{*}\right)$ of the upper 
level MO-A-FP problem should be determined first. We consider in this paper the FGP approach of C. T. Chang [9] that solve fractional programming problem with absolute-value function, to solving the first-level of problem. In section 5, the FGP model of Chang for solving the ULDM problem, is presented to facilitate the achievement of $X^{*}=\left(X_{1}^{*}, X_{2}^{*}\right)$.

Let $t_{k}^{L}$ and $t_{k}^{R}\left(k=1,2, \ldots, n_{1}\right)$ be the maximum acceptable negative and positive tolerance values on the decision vector considered by the ULDM. This is a triangular fuzzy member [10]. The tolerance give the lower level DMs an extent feasible region to search for the satisfactory solution. The linear membership functions for the decision vector $X_{1}=\left(x_{1}^{1}, x_{1}^{2}, \ldots, x_{1}^{n_{1}}\right)$ controlled by the ULDM can be formulated as

$$
\begin{gathered}
\mu_{x_{1}^{k}}\left(x_{1}^{k}\right)=\left\{\begin{array}{lr}
\frac{x_{1}^{k}-\left(x_{1}^{k *}-t_{k}^{L}\right)}{t_{k}^{L}} & x_{1}^{k *}-t_{k}^{L} \leq x_{1}^{k} \leq x_{1}^{k *} \\
\frac{\left(x_{1}^{k *}+t_{k}^{R}\right)-x_{1}^{k}}{t_{k}^{k}} & x_{1}^{k *} \leq x_{1}^{k} \leq x_{1}^{k *}+t_{k}^{R} \\
0 & \text { otherrwise }
\end{array}\right. \\
k=1,2, \ldots, n_{1} .
\end{gathered}
$$

It is mentioned that the tolerance $t_{k}^{L}$ and $t_{k}^{R}$ are not necessarily same. Also the DM may desire to shift the range of $x_{k}$. Following Pramanik and Roy [11] and Sinha [12], this shift can be achieved. In decision making situation, the aim of each DM is to achieve highest membership value (unity) of the associated fuzzy goal in order to obtain the absolute satisfactory solution. However, in real practice, achievement of all membership values to the highest degree (unity) is not possible due to conflicting objectives. Therefore, decision policy for minimizing the regrets of the DMs for all the levels should be taken into consideration. Hence, each DM should try to maximize his or her membership function by making them as close as possible to unity by minimizing its negative-and positive-deviational variables. Therefore, in effect, we are simultaneously optimizing all the objective functions. So, for the defined membership functions in (3) and (4), the flexible membership goals having the aspired level unity can be represented as

$$
\begin{gathered}
\mu_{f_{i j}}\left(f_{i j}\left(X_{1}, X_{2}\right)\right)+d_{i j}^{-}-d_{i j}^{+}=1, \quad i=1,2, \quad j=1,2, \ldots, m_{i}, \\
\mu_{x_{1}^{k}}\left(x_{1}^{k}\right)+d_{k}^{-}-d_{k}^{+}=1, \quad k=1,2, \ldots n_{1},
\end{gathered}
$$

or equivalently as

$$
\begin{aligned}
& \frac{\overline{u_{i j}}-f_{i j}\left(X_{1}, X_{2}\right)}{\bar{u}_{i j}-\bar{l}_{i j}}+d_{i j}^{-}-d_{i j}^{+}=1, \quad i=1,2, \quad j=1,2, \ldots, m_{i}, \\
& \frac{x_{1}^{k}-\left(x_{1}^{k *}-t_{k}^{L}\right)}{t_{k}^{L}}+d_{k}^{L-}-d_{k}^{L+}=1, \quad k=1,2, \ldots n_{1},
\end{aligned}
$$




$$
\frac{\left(x_{1}^{k *}+t_{k}^{R}\right)-x_{1}^{k}}{t_{k}^{R}}+d_{k}^{R-}-d_{k}^{R+}=1, \quad k=1,2, \ldots n_{1},
$$

here $d_{k}^{-}=\left(d_{k}^{L-}, d_{k}^{R-}\right), d_{k}^{+}=\left(d_{k}^{L+}, d_{k}^{R+}\right)$ and $d_{i j}^{-}, d_{k}^{L-}, d_{k}^{R-}, d_{i j}^{+}, d_{k}^{L+}, d_{k}^{R+} \geq 0$ with $d_{i j}^{-} \times d_{i j}^{+}=0, i=1,2, \quad j=1,2, \ldots, m_{i}, d_{k}^{L-} \times d_{k}^{L+}=0$ and $d_{k}^{R-} \times d_{k}^{R+}=0$, $k=1,2, \ldots n_{1}$, represent the under-and over-deviational, respectively, from the aspired levels. Now, FGP approach to BLMO-A-FP problem can be presented as:

$$
\begin{gathered}
\operatorname{MinZ}=\sum_{j=1}^{m_{1}} w_{1 j}\left(d_{1 j}^{-}+d_{1 j}^{+}\right)+\sum_{k=1}^{n_{1}}\left[w_{k}^{L}\left(d_{k}^{L+}+d_{k}^{L-}\right)+w_{k}^{R}\left(d_{k}^{R+}+d_{k}^{R-}\right)\right] \\
+\sum_{j=1}^{m_{2}} w_{2 j}\left(d_{2 j}^{-}+d_{2 j}^{-}\right)
\end{gathered}
$$

subject to

$$
\begin{aligned}
& \frac{\overline{u_{i j}}-f_{i j}\left(X_{1}, X_{2}\right)}{\bar{u}_{i j}-\bar{l}_{i j}}+d_{i j}^{-}-d_{i j}^{+}=1, \quad i=1,2, \quad j=1,2, \ldots, m_{i}, \\
& \frac{x_{1}^{k}-\left(x_{1}^{k *}-t_{k}^{L}\right)}{t_{k}^{L}}+d_{k}^{L-}-d_{k}^{L+}=1, \quad k=1,2, \ldots n_{1} \\
& \frac{\left(x_{1}^{k *}+t_{k}^{R}\right)-x_{1}^{k}}{t_{k}^{R}}+d_{k}^{R-}-d_{k}^{R+}=1, \quad k=1,2, \ldots n_{1} \\
& X \in S, \quad X \quad \text { is unrestricted. }
\end{aligned}
$$

In the present formulation, numerical weights $w_{i j},\left(i=1,2, j=1,2, \ldots, m_{i}\right)$ $w_{k}^{L}$ and $w_{k}^{R}\left(k=1,2, \ldots n_{1}\right)$ are determined as [4]

$$
\begin{aligned}
& w_{i j}=\frac{1}{\overline{u_{i j}}-\bar{l}_{i j}} \quad i=1,2, \quad j=1,2, \ldots, m_{i}, \\
& w_{k}^{L}=\frac{1}{t_{k}^{L}}, \quad w_{k}^{R}=\frac{1}{t_{k}^{R}}, \quad k=1,2, \ldots n_{1} .
\end{aligned}
$$

\section{Linearization of Membership Goals}

The $i j \operatorname{th}\left(i=1,2, j=1,2, \ldots, m_{i}\right)$ membership goal in (6) can be presented as

$$
h_{i j} \overline{u_{i j}}-h_{i j} f_{i j}\left(X_{1}, X_{2}\right)+d_{i j}^{-}-d_{i j}^{+}=1 \quad \text { where } \quad h_{i j}=\frac{1}{\overline{u_{i j}}-\overline{l_{i j}}} .
$$

Introducing the expression of $f_{i j}\left(X_{1}, X_{2}\right)$ from (2). The above goal can be presented as

$$
h_{i j} \overline{u_{i j}}-h_{i j}\left(\frac{\alpha_{i j}+\sum_{k=1}^{n} c_{i k}\left|x_{k}\right|}{\beta_{i j}+\sum_{k=1}^{n} d_{i k}\left|x_{k}\right|}\right)+d_{i j}^{-}-d_{i j}^{+}=1,
$$


or equivalently as

$$
\begin{gathered}
-h_{i j}\left(\alpha_{i j}+\sum_{k=1}^{n} c_{i k}\left|x_{k}\right|\right)+d_{i j}^{-}\left(\beta_{i j}+\sum_{k=1}^{n} d_{i k}\left|x_{k}\right|\right)-d_{i j}^{+}\left(\beta_{i j}+\sum_{k=1}^{n} d_{i k}\left|x_{k}\right|\right) \\
=\left(1-h_{i j} \overline{u_{i j}}\right)\left(\beta_{i j}+\sum_{k=1}^{n} d_{i k}\left|x_{k}\right|\right) .
\end{gathered}
$$

Hence we have

$$
\begin{aligned}
\left(-h_{i j} c_{i k}-L_{i j}^{\circ} d_{i k}\right) \sum_{k=1}^{n}\left|x_{k}\right| & +d_{i j}^{-}\left(\beta_{i j}+\sum_{k=1}^{n} d_{i k}\left|x_{k}\right|\right)-d_{i j}^{+}\left(\beta_{i j}+\sum_{k=1}^{n} d_{i k}\left|x_{k}\right|\right) \\
& =L_{i j}^{\circ} \beta_{i j}+h_{i j} \alpha_{i j}
\end{aligned}
$$

where $L_{i j}^{\circ}=1-h_{i j} \overline{u_{i j}}$.

Letting $D_{i j}^{-}=d_{i j}^{-}\left(\beta_{i j}+\sum_{k=1}^{n} d_{i k}\left|x_{k}\right|\right), D_{i j}^{+}=d_{i j}^{+}\left(\beta_{i j}+\sum_{k=1}^{n} d_{i k}\left|x_{k}\right|\right), C_{i j}=$ $-h_{i j} c_{i k}-L_{i j}^{\circ} d_{i k}$ and $G_{i j}=L_{i j}^{\circ} \beta_{i j}+h_{i j} \alpha_{i j}$, then the form of the expression in (8) is obtained as

$$
C_{i j} \sum_{k=1}^{n}\left|x_{k}\right|+D_{i j}^{-}-D_{i j}^{+}=G_{i j},
$$

with $D_{i j}^{-}, D_{i j}^{+} \geq 0$ and $D_{i j}^{-} \times D_{i j}^{+}=0$ since $d_{i j}^{-}, d_{i j}^{+} \geq 0$ and $\beta_{i j}+\sum_{k=1}^{n} d_{i k}\left|x_{k}\right|>0$. Clearly, when a membership goal is fully achieved, $d_{i j}^{-}=0$ and its achievement is zero, $d_{i j}^{-}=1$ are found in the solution. So, involvement of $d_{i j}^{-} \leq 1$ in the solution leads to impose the following constraint to the model of the problem

$$
\frac{D_{i j}^{-}}{\beta_{i j}+\sum_{k=1}^{n} d_{i k}\left|x_{k}\right|} \leq 1
$$

i.e.

$$
-\sum_{k=1}^{n} d_{i k}\left|x_{k}\right|+D_{i j}^{-} \leq \beta_{i j} .
$$

Next, we for linearize the absolute term $d_{i k}\left|x_{k}\right|$ that can be expressed as follows:

program $A$ :

$$
\begin{gathered}
\text { Minimize } d_{i k}\left|x_{k}\right|, \\
d_{i k}\left|x_{k}\right|= \begin{cases}d_{i k} x_{k} & x_{k} \geq 0 \\
-d_{i k} x_{k} & x_{k} \leq 0\end{cases}
\end{gathered}
$$


using Program B as follows:

program $B$ :

$$
\begin{array}{cl}
\min & b_{k} x_{k}+\left(b_{k}-1\right) x_{k} \\
\text { subject } & \text { to } \quad\left(b_{k}-1\right) x_{k} \geq 0 \\
& b_{k} x_{k} \geq 0,
\end{array}
$$

where $b_{k}\left(k=1,2, \ldots, n_{1}\right)$ are binary variables.

Program A and Program B are equivalent in the sense that they have the same optimal solution [9]. Also the quadratic mixed binary term $b_{k} x_{k}$ in program B can be linearized of the Ref. [13]. Therefore, under the framework of minsum GP, the equivalent proposed FGP model of problem (6) becomes

$$
\begin{aligned}
\operatorname{MinZ}=\sum_{j=1}^{m_{1}} w_{1 j}\left(D_{1 j}^{-}+\right. & \left.D_{1 j}^{+}\right)+\sum_{k=1}^{n_{1}}\left[w_{k}^{L}\left(d_{k}^{L+}+d_{k}^{L-}\right)+w_{k}^{R}\left(d_{k}^{R+}+d_{k}^{R-}\right)\right] \\
& +\sum_{j=1}^{m_{2}} w_{2 j}\left(D_{2 j}^{-}+D_{2 j}^{-}\right)
\end{aligned}
$$

subject to

$$
\begin{aligned}
& C_{1 j} \sum_{k=1}^{n}\left|x_{k}\right|+D_{1 j}^{-}-D_{1 j}^{+}=G_{1 j}, \quad j=1,2, \ldots, m_{1} \\
& C_{2 j} \sum_{k=1}^{n}\left|x_{k}\right|+D_{2 j}^{-}-D_{2 j}^{+}=G_{2 j}, \quad j=1,2, \ldots, m_{2} \\
& \frac{\left(x_{1}^{k *}+t_{k}^{R}\right)-x_{1}^{k}}{t_{k}^{R}}+d_{k}^{R-}-d_{k}^{R+}=1, \quad k=1,2, \ldots n_{1} \\
& \frac{x_{1}^{k}-\left(x_{1}^{k *}-t_{k}^{L}\right)}{t_{k}^{L}}+d_{k}^{L-}-d_{k}^{L+}=1, \quad k=1,2, \ldots n_{1} \\
& -\sum_{k=1}^{n} d_{i k}\left|x_{k}\right|+D_{i j}^{-} \leq \beta_{i j}, \quad i=1,2, \quad j=1,2, \ldots, m_{i} \\
& -\sum_{k=1}^{n} d_{i k}\left|x_{k}\right|+D_{i j}^{+} \leq \beta_{i j}, \quad i=1,2, \quad j=1,2, \ldots, m_{i} \\
& X \in S^{2} \quad X \quad \text { is unrestricted. } \\
& D_{i j}^{-}, D_{i j}^{+} \geq 0, \quad i=1,2, \quad j=1,2, \ldots, m_{i} \\
& d_{k}^{L-}, d_{k}^{L+} \geq 0 \text { with } d_{k}^{L-} \times d_{k}^{L+}=0, \quad k=1,2, \ldots, n_{1}, \\
& d_{k}^{R-}, d_{k}^{R+} \geq 0 \text { with } d_{k}^{R-} \times d_{k}^{R+}=0, \quad k=1,2, \ldots, n_{1} .
\end{aligned}
$$

The FGP model (13) provides the most satisfactory decision for both the ULDM and the LLDM by achieving the aspired levels of the membership goals to the extent possible in the decision environment. The solution procedure is straightforward and illustrated via the following example. 


\section{FGP Model for ULDM Problem}

In this section, the FGP model of Chang [9], for solving the first-level MOFP problem with absolute-value function, is presented here to facilitate the achievement of $X^{*}=\left(X_{1}^{*}, X_{2}^{*}\right)$. This solution is used to elicit the membership functions of the decision vectors $X_{1}=\left(x_{1}^{1}, x_{1}^{2}, \ldots, x_{1}^{n_{1}}\right)$, that included in the FGP approach for solving BLMO-A-FP problem that proposed in this article.

The ULDM problem is

$$
\begin{aligned}
& \min F_{1}\left(X_{1}, X_{2}\right)=\min \left(f_{11}\left(X_{1}, X_{2}\right), f_{12}\left(X_{1}, X_{2}\right), \ldots, f_{1 m_{1}}\left(X_{1}, X_{2}\right)\right) \\
& \text { subject to } \\
& X \in S=\left\{X=\left(X_{1}, X_{2}\right) \in R^{n} \mid A_{1} X_{1}+A_{2} X_{2}\left(\begin{array}{c}
\leq \\
= \\
\geq
\end{array}\right) b, b \in R^{m}\right\} \neq \emptyset
\end{aligned}
$$

And the FGP model of Chang [9] can be rewritten as

$$
\min Z=\sum_{j=1}^{m_{1}} w_{1 j}\left(D_{1 j}^{-}+D_{1 j}^{+}\right)
$$

subject to

$$
\begin{array}{ll}
C_{1 j} \sum_{k=1}^{n}\left|x_{k}\right|+D_{1 j}^{-}-D_{1 j}^{+}=G_{1 j}, \quad & j=1,2, \ldots, m_{1} \\
-\sum_{k=1}^{n} d_{i k}\left|x_{k}\right|+D_{1 j}^{-} \leq \beta_{1 j}, & j=1,2, \ldots, m_{1} \\
-\sum_{k=1}^{n} d_{1 k}\left|x_{k}\right|+D_{1 j}^{+} \leq \beta_{1 j}, & j=1,2, \ldots, m_{1} \\
X \in S, \quad X \quad \text { is unrestricted, } & \\
D_{1 j}^{-}, D_{1 j}^{+} \geq 0, \quad j=1,2, \ldots, m_{1} . &
\end{array}
$$

\section{Numerical Example}

To demonstrate the solution method for BLMO-A-FP, let consider the following example.

(Upper Level)

$$
\min _{X_{1}}\left(f_{11}:-1 \leq \frac{\left|x_{1}\right|+\left|x_{2}\right|-6}{\left|x_{1}\right|+\left|x_{2}\right|+4} \leq 1, f_{12}: 0 \leq \frac{2\left|x_{1}\right|+\left|x_{2}\right|-1}{\left|x_{2}\right|+4} \leq 2\right)
$$


where $X_{2}$ solves

(Lower Level)

$$
\begin{gathered}
\min _{X_{2}}\left(f_{21}: 1 \leq \frac{\left|x_{1}\right|+\left|x_{2}\right|+2}{2\left|x_{1}\right|+\left|x_{2}\right|} \leq 3, f_{22}: 0 \leq \frac{\left|x_{1}\right|+3\left|x_{2}\right|}{2\left|x_{1}\right|+\left|x_{2}\right|+1} \leq 3,\right. \\
\left.f_{23}:-4 \leq \frac{-4\left|x_{1}\right|+2\left|x_{2}\right|}{\left|x_{1}\right|+\left|x_{2}\right|} \leq 2\right)
\end{gathered}
$$

subject to

$$
\begin{aligned}
& -x_{1}+x_{2} \leq-1 \\
& 2 x_{1}-x_{2} \leq 10 \\
& -2 x_{1}-x_{2} \leq 8
\end{aligned}
$$

Now, based on (5) the membership goals of ULDM can be expressed as

$$
\begin{aligned}
& \mu_{f_{11}}\left(f_{11}\left(x_{1}, x_{2}\right)\right)=\frac{1-\frac{\left|x_{1}\right|+\left|x_{2}\right|-6}{\left|x_{1}\right|+\left|x_{2}\right|+4}}{2}+d_{11}^{-}-d_{11}^{+}=1, \\
& \mu_{f_{12}}\left(f_{12}\left(x_{1}, x_{2}\right)\right)=\frac{2-\frac{2\left|x_{1}\right|+\left|x_{2}\right|-1}{\left|x_{2}\right|+4}}{2}+d_{12}^{-}-d_{12}^{+}=1,
\end{aligned}
$$

Also, the membership goals of LLDM can be expressed as

$$
\begin{aligned}
& \mu_{f_{21}}\left(f_{21}\left(x_{1}, x_{2}\right)\right)=\frac{3-\frac{\left|x_{1}\right|+\left|x_{2}\right|+2}{2\left|x_{1}\right|+\left|x_{2}\right|}}{2}+d_{21}^{-}-d_{21}^{+}=1, \\
& \mu_{f_{22}}\left(f_{22}\left(x_{1}, x_{2}\right)\right)=\frac{3-\frac{\left|x_{1}\right|+3\left|x_{2}\right|}{2\left|x_{1}\right|+\left|x_{2}\right|+1}}{3}+d_{22}^{-}-d_{22}^{+}=1, \\
& \mu_{f_{23}}\left(f_{23}\left(x_{1}, x_{2}\right)\right)=\frac{2-\frac{-4\left|x_{1}\right|+2\left|x_{2}\right|}{\left|x_{1}\right|+\left|x_{2}\right|}}{6}+d_{23}^{-}-d_{23}^{+}=1,
\end{aligned}
$$

Table 1 summarizes the coefficients $\alpha_{i j}, \beta_{i j}, c_{i k}$ and $d_{i k}$ for the first- and second-level objectives of the BLMO-A-FP problem. The upper and lower bound to the objective functions are also mentioned. The values $h_{i j}, L_{i j}^{\circ}, C_{i j}, G_{i j}$ and the weights $w_{i j}$ are calculated and also contained in the table.

First, the ULDM solves his/her problem based on (14) as follows:

$$
\min \quad 0.5 D_{11}^{-}+0.5 D_{11}^{+}+0.5 D_{12}^{-}+0.5 D_{12}^{+}
$$

subject to

$$
-\left|x_{1}\right|-\left|x_{2}\right|+D_{11}^{-}-D_{11}^{+}=-1
$$


Bi-Level Multi-Objective Absolute-Value Fractional programming Problems... 351

Table 1: Coefficients objective functions for the BLMO-A-FP problem

\begin{tabular}{cccccc}
\hline & $f_{11}$ & $f_{12}$ & $f_{21}$ & $f_{22}$ & $f_{23}$ \\
\hline$\alpha_{i j}$ & -6 & -1 & 2 & 0 & 0 \\
$\beta_{i j}$ & 4 & 4 & 0 & 1 & 0 \\
$c_{i j}$ & $(1,1)$ & $(2,1)$ & $(1,1)$ & $(1,3)$ & $(-4,2)$ \\
$d_{i j}$ & $(1,1)$ & $(0,1)$ & $(2,1)$ & $(2,1)$ & $(1,1)$ \\
$\bar{u}_{i j}$ & 1 & 2 & 3 & 3 & 2 \\
$\bar{l}_{i j}$ & -1 & 0 & 1 & 0 & -4 \\
$h_{i j}$ & 0.5 & 0.5 & 0.5 & 0.33 & 0.167 \\
$L_{i j}^{\circ}$ & 0.5 & 0 & -0.5 & 0 & 0.67 \\
$C_{i j}$ & $(-1,-1)$ & $(-1,-0.5)$ & $(0.5,0)$ & $(-0.33,-1)$ & $(0 .-1)$ \\
$G_{i j}$ & -1 & -0.5 & 1 & 0 & 0 \\
$w_{i j}$ & 0.5 & 0.5 & 0.5 & 0.33 & 0.167 \\
\hline \multicolumn{7}{c}{} & & & &
\end{tabular}

$$
\begin{aligned}
-\left|x_{1}\right|-0.5\left|x_{2}\right|+D_{12}^{-}-D_{12}^{+} & =-0.5 \\
-\left|x_{1}\right|-\left|x_{2}\right|+D_{11}^{-} & \leq 4 \\
-\left|x_{1}\right|-\left|x_{2}\right|+D_{11}^{+} & \leq 4 \\
-\left|x_{2}\right|+D_{12}^{-} & \leq 4 \\
-\left|x_{2}\right|+D_{12}^{+} & \leq 4 \\
-x_{1}+x_{2} & \leq-1 \\
2 x_{1}-x_{2} & \leq 10 \\
-2 x_{1}-x_{2} & \leq 8
\end{aligned}
$$

where absolute terms in above can be linearized using problem B. The software LINGO (ver. 11.0) is used to solve the problem. Optimal solution of the problem is $\left(x_{1}^{*}, x_{2}^{*}\right)=(0,-1)$. Let the ULDM decide $x_{1}^{*}=0$ with the negative and positive tolerance $t_{1}^{R}=t_{1}^{L}=0.4$.

Then, by using (13) the LLDM solves the following problem as follows:

$$
\begin{gathered}
\min \quad 0.5\left(D_{11}^{-}+D_{11}^{+}\right)+0.5\left(D_{12}^{-}+D_{12}^{+}\right)+0.5\left(D_{21}^{-}+D_{21}^{+}\right)+0.33\left(D_{22}^{-}+D_{22}^{+}\right) \\
\quad+0.167\left(D_{23}^{-}+D_{23}^{+}\right)+2.5\left(d_{1}^{L-}+d_{1}^{L+}\right)+2.5\left(d_{1}^{R-}+d_{1}^{R+}\right)
\end{gathered}
$$

subject to

$$
\begin{gathered}
-\left|x_{1}\right|-\left|x_{2}\right|+D_{11}^{-}-D_{11}^{+}=-1 \\
-\left|x_{1}\right|-0.5\left|x_{2}\right|+D_{12}^{-}-D_{12}^{+}=-0.5 \\
0.5\left|x_{1}\right|+D_{21}^{-}-D_{21}^{+}=1
\end{gathered}
$$




$$
\begin{aligned}
-0.33\left|x_{1}\right|-\left|x_{2}\right|+D_{22}^{-}-D_{22}^{+} & =0 \\
-\left|x_{2}\right|+D_{23}^{-}-D_{23}^{+} & =0 \\
-\left|x_{1}\right|-\left|x_{2}\right|+D_{11}^{-} & \leq 4 \\
-\left|x_{1}\right|-\left|x_{2}\right|+D_{11}^{+} & \leq 4 \\
-\left|x_{2}\right|+D_{12}^{-} & \leq 4 \\
-\left|x_{2}\right|+D_{12}^{+} & \leq 4 \\
-2\left|x_{1}\right|-\left|x_{2}\right|+D_{21}^{-} & \leq 0 \\
-2\left|x_{1}\right|-\left|x_{2}\right|+D_{21}^{+} & \leq 0 \\
-2\left|x_{1}\right|-\left|x_{2}\right|+D_{22}^{-} & \leq 1 \\
-2\left|x_{1}\right|-\left|x_{2}\right|+D_{22}^{+} & \leq 1 \\
-\left|x_{1}\right|-\left|x_{2}\right|+D_{23}^{-} & \leq 0 \\
-\left|x_{1}\right|-\left|x_{2}\right|+D_{23}^{+} & \leq 0 \\
2.5 x_{1}+d_{1}^{L-}-d_{1}^{L+} & =0 \\
-2.5 x_{1}+d_{1}^{R-}-d_{1}^{R+} & =0 \\
-x_{1}+x_{2} & \leq-1 \\
2 x_{1}-x_{2} & \leq 10 \\
-2 x_{1}-x_{2} & \leq 8
\end{aligned}
$$

where absolute terms in above can be linearized using problem B. The software LINGO (ver. 11.0) is used to solve the problem. Optimal solution of the problem is $\left(x_{1}, x_{2}\right)=(1,0)$ with objective functions values $f_{11}=-1, f_{12}=$ $0.25, f_{21}=1.5, f_{22}=0.33$ and $f_{23}=-4$, with membership functions values $\mu_{11}=1, \mu_{12}=0.87, \mu_{21}=0.75, \mu_{22}=0.88$ and $\mu_{23}=1$. Therefore we realize that $f_{11}$ and $f_{23}$ has reached the goal exactly, $f_{12}$ has 0.87 achieved, $f_{21}$ has 0.75 achieved and $f_{22}$ has 0.88 achieved.

\section{Conclusion}

This paper studies a bi-level multi-objective absolute-value fractional programming problem with fuzzy goal programming approach. We have extended the absolute-value fractional programming technique to bi-level multi-objective absolute-value fractional programming problem. It can be further verified that the constraints can be put in the form of absolute-value functions. 
Bi-Level Multi-Objective Absolute-Value Fractional programming Problems... 353

\section{References}

[1] W. Candler, R. Townsley, A linear two-level programming problem, Computer and Operations Research 9 (1982) 59-76.

[2] A. Charnes, W.W. Cooper, Management Models of Industrial Applications of Linear Programming (Appendix B), John Wiley and Sons, New York Vol. 1(1961).

[3] W. T. Lin, A survey of goal programming applications. Omega 8 (1980) $115-117$.

[4] R. H. Mohamed, The relationship between goal programming and fuzzy programming, Fuzzy Sets and Systems, 89(1997), 215-222.

[5] B.B. Pal, B.N Moitra, A goal programming procedure for multiple objective fuzzy linear fractional programming problem, Applicable Mathematics Its Perspectices and Challenges, Narosa Pub, New Delhi (2000) $347-362$.

[6] B.B Pal, B.N Moitra, A goal programming procedure for solving problems with multiple fuzzy goals using dynamic programming , European Journal of Operational Research, (2001)

[7] M. A. Abo-Sinha, A bi-level non-linear multi-objective decision making under fuzziness, Operation Research Society of India (OPSEARCH), $38(5)(2001) 484-495$.

[8] I. A. Baky, Fuzzy goal programming algorithm for solving decentralized bi-level multi-objective programming problems, Fuzzy Sets Systems, 160(2009) 2701-2713.

[9] C. T. Chang, Fractional programming with absolute-value functions: a fuzzy goal programming approach, Applied Mathematics and Computation $167(2005)$ 508-515

[10] Y.J. Lai, C.L. Hwang, Fuzzy Mathematical ProgrammingMethods and Applications, Springer, Berlin, 1993.

[11] S. Pramanik, T. Kumar Roy, Fuzzy goal programming approach to multilevel programming problems, European Journal of Operational Research 176 (2006) 1151-1166.

[12] S. Sinha, Fuzzy programming approach to multi-level programming problems, Fuzzy Sets and Systems 136 (2003) 189-202. 
[13] C. T. Chang, An eficient linearization approach for mixedinteger problems, European Journal of Operational Research 123 (2000) 652-659. 